\section{Learning by Doing: Applying the Concept of Pollen Viability in a Horticulture Classroom}

\author{
Kauahi Perez ${ }^{1}$
}

\begin{abstract}
ADDITIONAL INDEX wORDs. hands-on, inquiry, in vitro pollen germination, plumeria

SUMMARY. Learning by doing plays a critical role in a learner's conceptual understanding. By actively engaging with a concept, students gain experience and develop an enduring understanding of the concept. The concept of pollen viability is a critical component in the field of plant breeding and can be used to explain various aspects of pollen quality. An inquiry activity was designed to expose undergraduate students in a horticulture course to the concept of pollen viability and its application. The entire class was tasked with collaborating to identify an in vitro germination medium optimized to germinate plumeria (Plumeria rubra) pollen. To determine optimum sucrose and $\mathrm{pH}$ concentrations of the medium, student groups were assigned treatments of pollen from two plumeria cultivars that were germinated in Brewbaker and Kwack media of differing sucrose and $\mathrm{pH}$ concentrations. Students calculated the percentage of germinated pollen and assessed pollen tube integrity and used these variables as evidence of an optimized medium. Although undergraduates were engaged in authentic research practices during the inquiry activity, lack of time and resources impeded completion of the activity. However, students were exposed to methods and instrumentation directly related to evaluating pollen viability. Moreover, they were exposed to the basic practice of pollen quality assessment that they can use to carry out investigations on pollen fertility. In addition, insight was gained to improve the inquiry activity in the future. Now, well-informed modifications to the inquiry activity can be made to pilot this activity in a formal horticulture laboratory section.
\end{abstract}

$\mathrm{W}$ e hear and we forget. We see and we remember. We do and we understand. This phrase touches on the importance of learning through experience. A concept related to this is called "learning by doing" (DuFour et al., 2006) and plays a pivotal role in students' understanding of a concept. When a concept is merely introduced in lecture or reading, students may regard it as something trivial and not bother internalizing it. When examples of how that concept is applied are given, students are more inclined to remember it. Through experience, students come to their own enduring understanding of the concept because they develop a working knowledge of how it is applied (Dewey, 1938). Thus, students are more likely to

Department of Tropical Plant and Soil Sciences, University of Hawaii at Manoa, 3190 Maile Way, Honolulu, HI 96822.

This paper was part of the workshop "Five-minute Impact Strategies for Engaging Students-Where to Start with Service-learning and Fundraising?" held on 4 Aug. 2015 at the ASHS Annual Conference, New Orleans, LA, and sponsored by the Teaching Methods (TCHG) Working Group. The original title of the presentation was "I Do...I Understand."

${ }^{1}$ Corresponding author. E-mail: bronsonp@hawaii. edu.

doi: 10.21273/HORTTECH03351-17 understand a concept if they have first-hand experience on how to apply a concept for themselves.

The concept of pollen viability is a critical component in the field of plant breeding and can be used to explain various phenomena of plant breeding as they relate to pollen quality. Pollen viability can be defined as the potential of a pollen grain to germinate, grow, and effect seed set (Dafni et al., 2005). Thus, the concept of pollen viability can be applied to infer the fertility of a given plant.

In vitro pollen germination is a common method used to evaluate pollen quality and is often employed in the fields of plant physiology, plant breeding, and pollination biology (Dafni et al., 2005). The process of in vitro pollen germination requires pollen grains to be suspended in a suitable in vitro medium, and allowed to germinate and grow pollen tubes. Data can be collected on the growth response of the pollen grains to infer pollen viability. Pollen viability is assessed by the percentage of germinated pollen grains in a sample and by the quality of pollen tubes. However, to accurately assess pollen viability, the optimum in vitro medium must first be identified.

Undergraduates in the Department of Tropical Plant and Soil Sciences (TPSS) horticulture program at the University of Hawaii at Manoa only receive brief exposure to the concept of pollen viability through lectures and assigned readings. They receive no hands-on experience to bolster their understanding on how to assess pollen viability, and thus often have a difficult time internalizing this crucial concept. Research supports that hands-on learning benefits students in terms of achievement and effective engagement with the content (Bauerle and Park, 2012). Building on this framework, a hands-on activity on pollen viability would enhance the learning experience of undergraduates. The objective of this paper is to describe an inquiry activity that included a major hands-on activity focused on the concept of pollen viability in an undergraduate horticulture course.

\section{Materials and methods}

COURSE BACKground. TPSS 300 Tropical Production Systems is a course that is taught each spring semester and has evolved to include a wider range of horticultural topics, more hands-on learning, and incorporates the use of graduate students to give lectures and lead laboratory activities (Kobayashi and Perez, 2009, 2010; Perez et al., 2011). The Spring 2015 course had 15 students and included of a mix of undergraduates from horticulture and other majors. The lecture period for which this activity was piloted lasts for $75 \mathrm{~min}$ and was taught by a graduate student as the guest instructor.

Pollen preparation. The day before class, pollen was germinated in Brewbaker and Kwack (BK) medium (Brewbaker and Kwack, 1963), with modifications of sucrose and $\mathrm{pH}$ concentrations (Table 1). Factorial combinations of three sucrose concentrations $(10 \%, 15 \%$, and $20 \%)$ and three levels of $\mathrm{pH}(5.0,5.5$, and 6.0) were prepared. Pollen grains from two plumeria cultivars, Keane and Tillie Hughes, were suspended in the different media on microscope slides. Cover slips were placed over microscope slides, and slides were labeled according to the corresponding 
Table 1. Treatment structure of Brewbaker and Kwack (1963) in vitro germination media used to germinate pollen of plumeria cvs. Keane and Tillie Hughes. Pollen was germinated at different sucrose [Sucrose (\%)] and pH concentrations in factorial combinations. Each microscope slide (Slide no.) contained one treatment, and slides were arranged in numbered petri dishes (Petri dish no.) according to the $\mathrm{pH}$ of the Brewbaker and Kwack media. Each petri dish represented a $\mathrm{pH}$ treatment. Each student group received one petri dish, and no treatments were replicated.

\begin{tabular}{|c|c|c|c|c|c|c|c|}
\hline \multicolumn{4}{|c|}{ Keane } & \multicolumn{4}{|c|}{ Tillie Hughes } \\
\hline Petri dish no. & Slide no. & $\mathrm{pH}$ & Sucrose (\%) & Petri dish no. & Slide no. & $\mathrm{pH}$ & Sucrose (\%) \\
\hline \multirow[t]{2}{*}{1} & 1 & 5.0 & 10 & 4 & 1 & 5.0 & 10 \\
\hline & 3 & 5.0 & 20 & & 3 & 5.0 & 20 \\
\hline \multirow[t]{2}{*}{2} & 1 & 5.5 & 10 & 5 & 1 & 5.5 & 10 \\
\hline & 2 & 5.5 & 15 & & 2 & 5.5 & 15 \\
\hline & 2 & 6.0 & 15 & & 2 & 6.0 & 15 \\
\hline & 3 & 6.0 & 20 & & 3 & 6.0 & 20 \\
\hline
\end{tabular}

treatments. Pollen grains were left to germinate in the dark overnight in covered petri dishes lined with moistened filter paper.

STARTER ACTIVITY. This activity was designed to give a brief overview about how pollen viability applies to plant breeding and genetics, and included a lecture and a hands-on component. The lecture component involved a 15 -min lecture to introduce the concept of pollen viability, and explain quantitative and qualitative methods used to assess pollen viability. Also discussed was how to evaluate in vitro pollen germination, including viewing examples of what good and bad in vitro pollen germination looked like when pollen was grown in optimal and suboptimal conditions, respectively.

The hands-on component allowed students to see, touch, and smell flowers of plumeria cultivars Keane and Tillie Hughes. Students also completed a brief question set regarding the horticultural importance of morphological and olfactory traits of these two cultivars. In addition, students dissected flowers to locate the pistils and stamens so they would notice that the structure of plumeria flowers are not like the commonly diagrammed flowers often illustrated in introductory textbooks. This hands-on starter activity was done so students could develop a more relevant learning experience and make connections for the upcoming investigation activity.

INQUIRY ACTIVITY. Students were given the task of collaborating as an entire class to identify the optimum medium for germinating plumeria pollen. Students were divided into groups of three and assigned to a station that contained a microscope, a differential cell counter (Clay-Adams, New York, NY), and a large petri dish containing three microscope slides with pregerminated pollen from one of the two plumeria cultivars. Each slide within a petri dish corresponded to a sucrose treatment, and each petri dish corresponded to a $\mathrm{pH}$ treatment.

After a demonstration of how to use the cell counter and score germinated pollen, student groups were given a guided activity sheet with specific questions that helped them to identify key quantitative and qualitative aspects of assessing pollen viability. For the quantitative component, student groups used cell counters to score a total of 100 germinated or nongerminated pollen grains from random fields of view on a microscope slide. This resulted in a percent of germinated pollen. For the qualitative aspect, students evaluated the pollen tube integrity of germinated pollen and checked for any pollen tube abnormalities. Because there were three slides per petri dish, each student in a group had the opportunity to evaluate pollen germinated in one of three sucrose treatments. On the basis of pollen germination percent and pollen quality, students had to decide which sucrose concentration resulted in optimum pollen germination.

Because each group recorded data on their guided activity sheet, individual groups would see that sucrose concentration affects pollen germination. As groups completed data collection, student data were used to generate graphs in real time of percent germinated pollen of individual cultivars by sucrose and $\mathrm{pH}$ treatment. The graphs were intended to show the effects of sucrose and $\mathrm{pH}$ on pollen germination and differences in response between cultivars.

\section{Results and discussion}

Some groups could not complete data collection before the end of the 75-min class, which was attributed to the fact that only three cell counters were available for groups to share. So when some groups were using the cell counters to quantify pollen viability, other groups evaluated the quality of pollen tubes as they waited to use the cell counters. In addition, students had logistical difficulties scanning microscope slides to count pollen while simultaneously operating the cell counter. Moreover, clumped pollen grains made it difficult for students to distinguish between germinated and nongerminated pollen grains. Because some groups could not collect all the data for their assigned treatments by the end of the period, data collection was completed by the guest instructor, and graphs of pollen response to treatment for each cultivar were generated.

INQUIRY ACTIVITY SYNTHESIS. Reflections through a synthesis allow learners to derive meaning from their experiences of an activity (Fiddler and Marienau, 2008). Thus, a presentation was developed as a "take home message" for the entire activity so students could reflect on their own understanding of the pollen viability 
concept. The synthesis presentation entailed a recap of the entire activity, a general interpretation of the results for the class, and pointed out pertinent learning outcomes from the activity. The students received this presentation via e-mail and were encouraged to respond via e-mail with questions and feedback. Although no students responded with questions or feedback, the course's lead instructor gave insightful feedback on the activity.

The highlight of this activity was creating an environment of experiential learning by having students learn by doing. Learning by doing helps to develop a deeper knowledge and greater commitment than learning by reading a textbook or listening to a lecture (DuFour et al., 2006). By having first-hand experience exploring how pollen viability is applied to assessing pollen fertility, students will better understand this concept.

Another goal of this activity was to engage students in the authentic research practices of data collection and making observations, and in doing so to take ownership of their learning. However, in a simple inquiry activity like this, rather than letting students develop their own questions to investigate, students were told what to investigate. Although ownership of learning may have been compromised, the time constraints and the diversity of student majors within this class did not allow for an authentic inquiry experience (Chinn and Malhotra, 2002). However, simple inquiry tasks, such as taking data and carrying out basic observations, are still elements of authentic research that can be useful to pique student interest in unfamiliar concepts such as pollen viability.

Limitations. There were several limitations to this pilot activity. One limitation was that none of the treatments were replicated. However, the focus of this activity was not exposing undergraduates to scientific methodology. Rather, the purpose was to give students an overview of pollen quality assessment, give students an idea of how real-world scientists test for this, show how to conduct a preliminary experiment, and to increase student interest in the field of plant breeding.

A second limitation was the time constraint of a 75-min lecture period.
A simple inquiry activity, as small and guided as this was, still took longer than expected. The 75-min period did not allow time for students to absorb the information learned and reflect on their findings, nor was there enough time for students to share their findings as a class. To create a more meaningful learning experience, this kind of activity would be better suited for a 3-h laboratory section.

Modifications. If this activity was incorporated into a 3-h laboratory section, a major modification to the activity would be toward logistics within student groups. To expedite the process of scoring pollen, students will be assembled into groups of three, and each member of the group will assume a role as a "caller," "counter," or "recorder." As the "caller" scans the microscope slide for germinated pollen, the student will verbally score the pollen grain in view by saying "dead" (nongerminated) or "alive" (germinated). The "counter" will operate the cell counter and will push a red or white button if the "caller" indicates a dead or live pollen grain, respectively. When the "counter" acquires scores of 100 pollen grains, the "recorder" will record the amount of live (germinated) pollen as indicated on the cell counter to obtain a percentage of germinated pollen grains. This process should be repeated until a total of 300 pollen grains have been scored. The "recorder" will then obtain an average of percent germination and report that to the instructor.

Furthermore, different aspects of this activity could be expanded for a more authentic inquiry experience for students. For instance, students could complete an investigation on comparing pollen viability between multiple cultivars, comparing different formulations of pollen germination media, or defining requirements of an optimum pollen germination medium.

This kind of exercise can also be used to exemplify the various applications of pollen quality assessments. For instance, students could carry out investigations to determine at what stage of flowering that pollen is most viable, whether a particular plant is a better pollen donor or receiver for breeding purposes, or how long the pollen of a particular species can be stored before its viability is compromised. In short, applying the concept of pollen viability can be used to understand different aspects of pollen fertility as it relates to plant breeding and genetics.

\section{Conclusion}

Learning by doing is a method employed to bolster a learner's conceptual understanding. Applying the concept of pollen viability was the intention of this inquiry activity. Although the entire activity could not be carried out to completion, students were exposed to methods and instrumentation that most undergraduates in our program do not experience. Moreover, students were given exposure to the basic practice of pollen quality assessment, a practice critical to determining pollen fertility. Because this was the first attempt at piloting this kind of activity, many lessons were learned. Well-informed modifications will be made to the activity before including it in a 3 -h laboratory section for TPSS 300 in Spring 2016.

\section{Literature cited}

Bauerle, T.L. and T.D. Park. 2012. Experiential learning enhances student knowledge retention in the plant sciences. HortTechnology 22:715-718.

Brewbaker, J.L. and B.H. Kwack. 1963. The essential role of calcium ion in pollen germination and pollen tube growth. Amer. J. Bot. 50(9):859-865.

Chinn, C.A. and B.A. Malhotra. 2002. Epistemologically authentic inquiry in schools: A theoretical framework for evaluating inquiry tasks. Sci. Educ. 86(2):175218.

Dafni, A., P.G. Kevan, and B.C. Husband. 2005. Practical pollination biology. Enviroquest, Cambridge, UK.

Dewey, J. 1938. Experience \& education. Simon \& Schuster, New York, NY.

DuFour, R., R. Dufour, R. Eaker, and T. Many. 2006. Learning by doing: A handbook for professional learning communities at work. Solution Tree, Bloomington, IN.

Fiddler, M. and C. Marienau. 2008. Developing habits of reflection for meaningful learning. New Dir. Adult Contin. Educ. 118:75-85.

Kobayashi, K.D. and K. Perez. 2009. Enhanced active learning and TA 


\section{WORKSHOP}

involvement in a production systems horticulture course. Proc. Hawaii Intl. Conf. Educ. p. 738-748. 2 Feb. 2016. $<$ http://www.hiceducation.org/ EDU2009.pdf>.

Kobayashi, K.D. and K. Perez. 2010. Utilizing graduate students' firsthand horticultural experience in an undergraduate production systems course. HortScience 45:S246 (abstr.).
Perez, K., K.D. Kobayashi, and P-F. Wu. 2011. Keeping up with the times: Enhancement of topics in a tropical production systems course. HortScience 46: S397 (abstr.). 